\title{
Entecavir+tenofovir vs. lamivudine/telbivudine+ adefovir in chronic hepatitis B patients with prior suboptimal response
}

Hyun Young Woo, ${ }^{1,2}$, Jun Yong Park ${ }^{3}$, Si Hyun Bae ${ }^{4}$, Chang Wook Kim4, Jae Young Jang ${ }^{5}$, Won Young Tak ${ }^{6}$, Dong Joon $\mathrm{Kim}^{7,8}$, In Hee Kim ${ }^{9}$, Jeong Heo ${ }^{1,2, *}$, and Sang Hoon $\mathrm{Ahn}^{3, *}$

${ }^{1}$ Department of Internal Medicine, College of Medicine, Pusan National University, Busan; ${ }^{2}$ Medical Research Institute, Pusan National University Hospital, Busan; ${ }^{3}$ Department of Internal Medicine, Yonsei University College of Medicine, Seoul; ${ }^{4}$ Department of Internal Medicine, College of Medicine, The Catholic University of Korea, Seoul; ${ }^{5}$ Department of Internal Medicine, Institute for Digestive Research, Digestive Disease Center, Soonchunhyang University College of Medicine, Seoul; ${ }^{6}$ Department of Internal Medicine, Kyungpook National University School of Medicine, Daegu; ${ }^{7}$ Department of Internal Medicine, Hallym University College of Medicine, Chuncheon; ${ }^{8}$ Institute for Liver and Digestive Diseases, Hallym University, Chuncheon; ${ }^{9}$ Department of Internal Medicine, Chonbuk National University Hospital, Chonbuk National University College of Medicine, Chonju, Korea

\section{Graphical Abstract}

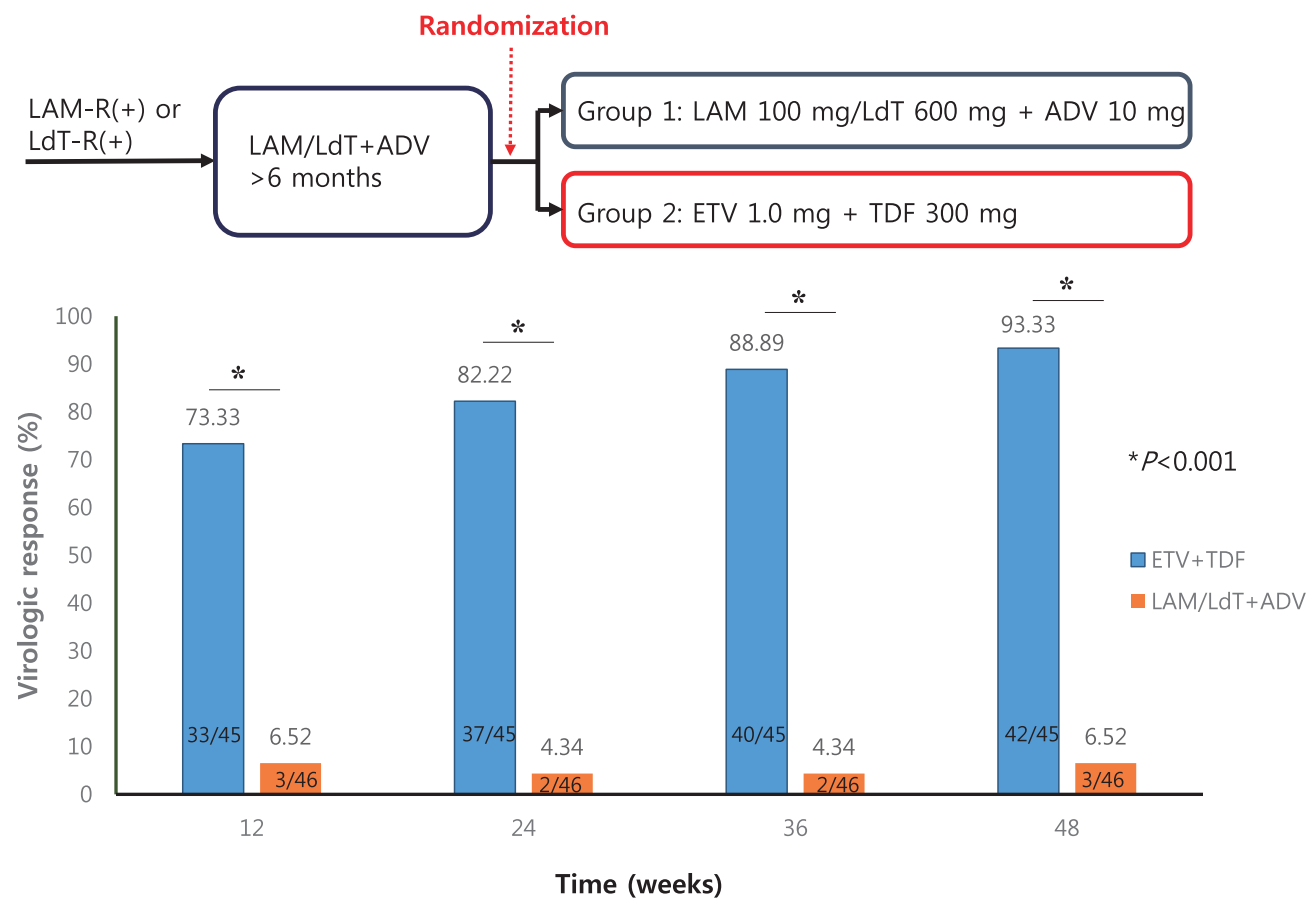

Copyright $(2020$ by Korean Association for the Study of the Liver

This is an Open Access article distributed under the terms of the Creative Commons Attribution Non-Commercial License (http://creativecommons.org/licenses/by-nc/3.0/) which permits unrestricted non-commercial use, distribution, and reproduction in any medium, provided the original work is properly cited. 
Background/Aims: Suboptimal responses to lamivudine or telbivudine plus adefovir (LAM/LdT+ADV) rescue therapy are common in patients with LAM-resistant hepatitis B virus (HBV) infections. We compared patients switched to entecavir plus tenofovir (ETV+TDF) to those maintained on LAM/LdT+ADV.

Methods: This prospective randomized controlled trial examined 91 patients whose serum HBV DNA levels were greater than $60 \mathrm{IU} / \mathrm{mL}$ after at least 24 weeks of treatment with LAM/LdT+ADV for LAM-resistant HBV. Patients were randomized to receive a new treatment $(E T V+T D F, n=45)$ or maintained on the same treatment (LAM/LdT+ADV, $n=46)$ for 48 weeks. Patients with baseline ADV resistance were excluded.

Results: Compared to LAM/LdT+ADV group, ETV+TDF group had more patients with a virologic response (42/45 [93.33\%] vs. $3 / 46[6.52 \%], P<0.001)$ and had a greater mean reduction in serum HBV DNA level from baseline $(-4.16$ vs. -0.37 $\left.\log _{10} \mathrm{IU} / \mathrm{mL}, P<0.001\right)$. Multivariate analysis indicated that high baseline HBV DNA level $(P=0.005)$ and LAM/LdT+ADV maintenance therapy $(P=0.001)$ were negatively associated with virologic response. At week 48 , additional ADV- or ETVassociated mutations were cleared in ETV+TDF group, but such mutations were present in $4.3 \%$ of patients in LAM/ LdT+ADV group $(P=0.106)$. The two groups had similar rates of adverse events.

Conclusions: ETV+TDF combination treatment led to a significantly higher rate of virologic response compared to LAM/ LdT+ADV combination treatment in patients with LAM-resistant HBV who had suboptimal responses to LAM/LdT+ADV regardless of HBV genotypic resistance profile (NCT01597934). (Clin Mol Hepatol 2020;26:352-363)

Keywords: Entecavir; Tenofovir; Lamivudine; Antiviral drug resistance; Adefovir

\section{Study Highlights}

This study showed that ETV+TDF led to a significantly higher rate of virologic response compared to LAM/LdT+ADV in patients with LAM-resistant HBV who had suboptimal responses to LAM/LdT+ADV regardless of the HBV genotypic resistance profile. Continuation of LAM/LdT+ADV afforded very little antiviral benefit, and increased the risk of RT mutations that confer drug resistance. The two treatment regimens had similar safety profiles.

\section{INTRODUCTION}

Patients with chronic hepatitis B (CHB) and continuously high levels of serum hepatitis B virus (HBV) DNA have an increased risk for progression of hepatic fibrosis and development of hepatocellular carcinoma (HCC). ${ }^{1,2}$ Current treatments for CHB aim to reduce the risk of hepatic events by providing complete virologic suppres- sion. $^{3-5}$ The introduction of nucleotide/nucleoside analogues (NUCs), which block the reverse transcription of HBV polymerase, has markedly improved the prognosis of these patients. ${ }^{6-9}$ However, drug resistance remains a clinical challenge when using antiviral therapies for CHB. The widespread use of antiviral agents with low genetic barriers to resistance, such as lamivudine (LAM), adefovir (ADV), telbivudine (LdT), and clevudine (approved in South

\begin{abstract}
Abbreviations:
ADV, adefovir; AEs, adverse events; ALT, alanine aminotransferase; AST, aspartate aminotransferase; CHB, chronic hepatitis B; ETV, entecavir; $H B e A g$, hepatitis B e antigen; HBsAg, hepatitis B surface antigen; HBV, hepatitis B virus; $\mathrm{HCC}$, hepatocellular carcinoma; LAM, lamivudine; LdT, telbivudine; NUCs, nucleotide/ nucleoside analogues; PCR, polymerase chain reaction; RT, reverse transcriptase; TDF, tenofovir; ULN, upper normal limit; YMDD, tyrosine-methionine-aspartic acid-aspartic acid

*These authors are equally contributed to this work as the corresponding author.
\end{abstract}

\section{Corresponding author: Sang Hoon Ahn}

Department of Internal Medicine, Yonsei University College of Medicine, 50-1 Yonsei-ro, Seodaemun-gu, Seoul 03722, Korea

Tel: +82-2-2228-1936, Fax: +82- 2-393-6884

E-mail:ahnsh@yuhs.ac

https://orcid.org/0000-0002-3629-4624

\section{Jeong Heo}

Department of Internal Medicine, College of Medicine, Pusan National University and Medical Research Institute, Pusan National University Hospital, 179 Gudeok-ro, Seo-Gu, Busan 49241, Korea

Tel: +82-51-240-7869, Fax: +82-51-244-8180

E-mail: jheo@pusan.ac.kr

https://orcid.org/0000-0003-0961-7851

Received : Dec. 18, 2019/ Revised : Apr. 6, 2020/ Accepted : Apr. 7, 2020 
Korea), as initial treatment is one of the main causes of the high prevalence of genotypic resistance to NUCs among patients with CHB in Asian countries. ${ }^{10}$ For example, patients taking LAM, the first approved oral nucleoside, have a $65 \%$ incidence of drug resistance after 5 years of treatment." Before the introduction of tenofovir (TDF) and entecavir (ETV), ADV was the only available recue therapy for patients with LAM resistance. However, sequential ADV monotherapy after the development of LAM resistance fails to achieve adequate virologic suppression in up to $25 \%$ of patients, and can also cause the development of genotypic resistance. ${ }^{12,13}$ Several studies reported that a substantial proportion of patients who were treated with LAM+ADV combination therapy developed persistently inadequate or suboptimal virologic responses, and that mutations in the tyrosine-methionine-aspartic acid-aspartic acid (YMDD) motif persisted despite rescue combination therapy. ${ }^{14,15}$ A suboptimal response to antiviral therapy can increase the risk of developing resistance to multiple NUCs, and also increase the risk of end-stage liver disease and HCC. ${ }^{16,17}$ Therefore, current guidelines suggest that the ideal treatment for CHB is to reduce the serum HBV DNA level to below the detection limit of real-time polymerase chain reaction (PCR). ${ }^{3,5}$

There is little consensus regarding the most effective antiviral therapy for patients with CHB who have suboptimal responses after LAM+ADV combination therapy. Before the approval of TDF, ETV+ADV was the most potent combination therapy for patients with $\mathrm{CHB}$ who had suboptimal responses to LAM+ADV. ${ }^{18}$ After its approval, TDF became an important and potent NUC used in antiviral regimens against CHB. In particular, TDF has high antiviral efficacy in patients with LAM resistance..$^{19}$ However, in patients who have failed to respond to LAM+ADV, previous research suggested that the efficacy of ETV or TDF monotherapy was inferior to that achieved in treatment-naïve patients. ${ }^{20-22}$ This emphasizes the need to identify the most effective combination therapy for treatment of multidrug-refractory CHB. Moreover, as patients with CHB require long-term antiviral therapy, they may develop resistance to treatments, even TDF-containing regimens. Combination treatment may be a better option than monotherapy to prevent further resistance in patients with LAM-resistant HBV. A recent retrospective study in South Korea showed the superior efficacy of ETV+TDF compared to ETV+ADV in patients with LAM-resistant $\mathrm{HBV}_{.}{ }^{23}$ No previous prospective studies have compared the efficacy of TDF+ETV with LAM/LdT+ADV in LAM-resistant patients who had suboptimal responses to LAM+ADV combination therapy. The present prospective study of patients with CHB with resistance to $L A M$ or $L d T$, who showed suboptimal responses to antiviral combination rescue therapy (LAM+ADV or LdT+ADV), compared the efficacy and safety of switching to TDF+ETV rather than maintaining LAM/LdT+ADV.

\section{MATERIALS AND METHODS}

\section{Study design}

This study was a randomized, open-label, prospective, multicenter trial of patients with infections by LAM- or LdT-resistant HBV (YMDD mutation) who had suboptimal responses to antiviral combination rescue therapy (LAM+ADV or LdT+ADV), and were receiving this therapy for at least 24 weeks. Patients were subjected to block randomization (1:1 ratio) and assigned to two treatment arms: 1) ETV (1.0 mg) plus TDF (300 mg) once daily or 2) LAM (100 mg) plus ADV ( $10 \mathrm{mg}$ ) or LdT $600 \mathrm{mg}$ plus ADV (10 $\mathrm{mg}$ ) once daily. The primary endpoint was measured at week 48 . At week 48 , further treatment with commercially available therapies performed was at the discretion of the investigator. During treatment, any patient who developed virologic breakthrough and showed alanine aminotransferase (ALT) flare (10-fold above the upper normal limit [ULN] of $40 \mathrm{IU} / \mathrm{mL}$ ) or liver decompensation was dropped out of the study and transitioned to commercially available antiviral therapy.

This study was conducted in accordance with the ethical principles of the Declaration of Helsinki 1975 (revised in 2000) and the regulatory requirements of all participating countries. Institutional approval was obtained at all clinical sites, and written informed consent was provided by all study participants. This study was registered at ClinicalTrials.gov under number NCT01597934 (https://clinicaltrials.gov/ct2/show/NCT01597934).

\section{Patients}

Patients with CHB, all of whom had detectable hepatitis B surface antigen (HBsAg) at screening and at 24 weeks or more before screening, were recruited from six hospital clinics in South Korea. Eligible patients were male or female, aged 20 years or older, hepatitis B e-antigen (HBeAg)-positive or -negative, and had compensated liver function (Child-Pugh class A). In addition, all patients had genotypic resistance to LAM or LdT (YMDD mutation) and were classified as suboptimal responders, defined as a $>1 \log _{10} \mathrm{IU} / \mathrm{mL}$ reduction in HBV DNA but with detectable HBV DNA (>60 IU/mL) after combination treatment with LAM 
(100 mg/day) plus ADV (10 mg/day) for at least 24 weeks that was ongoing at the time of randomization.

Patients with any of the following characteristics were excluded: history of genotypic resistance to ADV; ALT level more than 10fold above the ULN; most recent treatment that was not LAM+ADV or LdT+ADV; coinfection with HCV or HIV; pregnant or lactating; long-term use of an immunosuppressant or agent associated with high risk of hepatic/renal toxicity; history of liver transplantation or planning for liver transplantation; diagnosis of a malignant tumor and receiving chemotherapy; history of HCC or evidence of HCC; renal insufficiency (creatinine clearance rate below $50 \mathrm{~mL} / \mathrm{min}$ based on the Cockcroft-Gault equation); liver disease other than CHB (e.g., hemochromatosis, Wilson's disease, alcoholic liver disease, nonalcoholic fatty liver disease); and history of hypersensitivity to a study drug.

\section{Outcome analyses}

The primary efficacy endpoint was virologic response, defined as an HBV DNA level below $60 \mathrm{IU} / \mathrm{mL}$ (approximately 300 copies $/ \mathrm{mL}$ ) based on real-time PCR measurements at week 48.

The secondary efficacy endpoints were: 1) HBV DNA level below $60 \mathrm{IU} / \mathrm{mL}$ at weeks 12, 24, and 36; 2) ALT normalization (below the ULN); 3) mean HBV DNA level at weeks 12, 24, 36, and 48; and 4) HBeAg loss, HBeAg seroconversion (among HBeAg positive patients), and HBsAg loss at weeks 24 and 48. Surveillance of HBV antiviral drug resistance was performed on all baseline samples and at weeks 12, 24, 36, and 48 if there was detectable HBV DNA.

After randomization, patients were evaluated at baseline and at weeks 12, 24, 36, and 48. At each visit, vital signs were recorded, physical examination was performed, and adverse events were recorded. Efficacy analyses were based on plasma HBV DNA level, liver biochemistry, and hematology (all measured at baseline and at weeks 12, 24, 36, and 48). Serological measurements of HBsAg, anti-HBs antibody, HBeAg, and anti-HBe antibody were performed at weeks 24 and 48 using a radioimmunoassay (Abbott Laboratories, Abbott Park, IL, USA).

Routine biochemical tests (ALT, aspartate aminotransferase [AST], albumin, total bilirubin, and serum creatinine) were performed using a Sequential Multiple Autoanalyzer. HBV DNA was measured using real-time PCR assay on a Cobas TaqMan 48 Analyzer (Roche Molecular Diagnostics, Branchburg, NJ, USA), which had a limit of detection of $20 \mathrm{IU} / \mathrm{mL}$. Genotypic resistance was determined using restriction fragment mass polymorphism. ${ }^{24}$
Adverse events (AEs) and safety-related clinical laboratory tests were recorded at every visit. All patients with AEs that led to discontinuation were recorded up to week 48.

\section{Statistical analysis}

Efficacy analyses were performed based on patients who received at least one dose of the study medication (modified intentto-treat analysis). Discontinuation of treatment prior to week 48 was considered treatment failure for the primary endpoint. Since there were no clinical data on ETV+TDF combination treatment at the time of this study, the projected response rates were based on a previous study ( $4 \%$ for LAM+ADV and $29 \%$ for ETV+ADV). ${ }^{25}$ Comparison of the number of patients who achieved the primary endpoint used two-sided Fisher's exact test. A sample size of 104 randomized patients (52 patients per treatment arm) was estimated to provide at least $90 \%$ power to detect a difference of up to $25 \%$ between the two groups, based on Fisher's exact test with a significance level of 0.05 and assuming a dropout rate of $20 \%$ over 1 year.

Variables were expressed as means with standard deviations or numbers and percentages. Between-group comparisons of continuous variables were determined using an independent $t$-test. Categorical variables were compared using either chi-square test or Fisher's exact test, as appropriate. Cumulative virologic response during treatment was calculated using the Kaplan-Meier method, and curves were compared using a log-rank test. Univariate and multivariate analyses were performed using logistic regression to identify the factors associated with virologic response at week 48. Factors with $P$-values $<0.2$ in univariate analysis, along with clinical factors found to be important in previous studies, were included in the multivariate analysis. A $P$-value below 0.05 was considered significant. All data were analyzed using SPSS (version 20.0; IBM, Somers, NY, USA).

\section{RESULTS}

\section{Patient population}

We initially randomized $116 \mathrm{HBV}$ patients, but excluded $25 \mathrm{pa}$ tients who were deemed ineligible. As a result, we ultimately enrolled 45 patients in ETV+TDF group and 46 patients in LAM/ LdT+ADV group (Fig. 1). At the end of this study (week 48), 89 patients received commercially available anti-HBV therapies; two 


\section{CLINCAL IndMOLECULAR
HEPATOLOGY}

patients in LAM/LdT+ADV group were lost to follow-up. The two groups had similar baseline characteristics (Table 1). More specifically, the two groups had similar rates of liver cirrhosis $(13.33 \%$ vs. $19.57 \%, P=0.423)$, HBeAg positivity ( $88.89 \%$ vs. $95.65 \%$,
$P=0.266)$, HBV DNA level (4.36 vs. $4.08 \log _{10} \mathrm{IU} / \mathrm{mL}, P=0.228$ ), and ALT level (27 vs. $32 \mathrm{U} / \mathrm{L}, P=0.422$ ). The two groups also had no significant differences in prior antiviral treatment regimens (Table 2) and genotypic resistance profiles (Table 3).

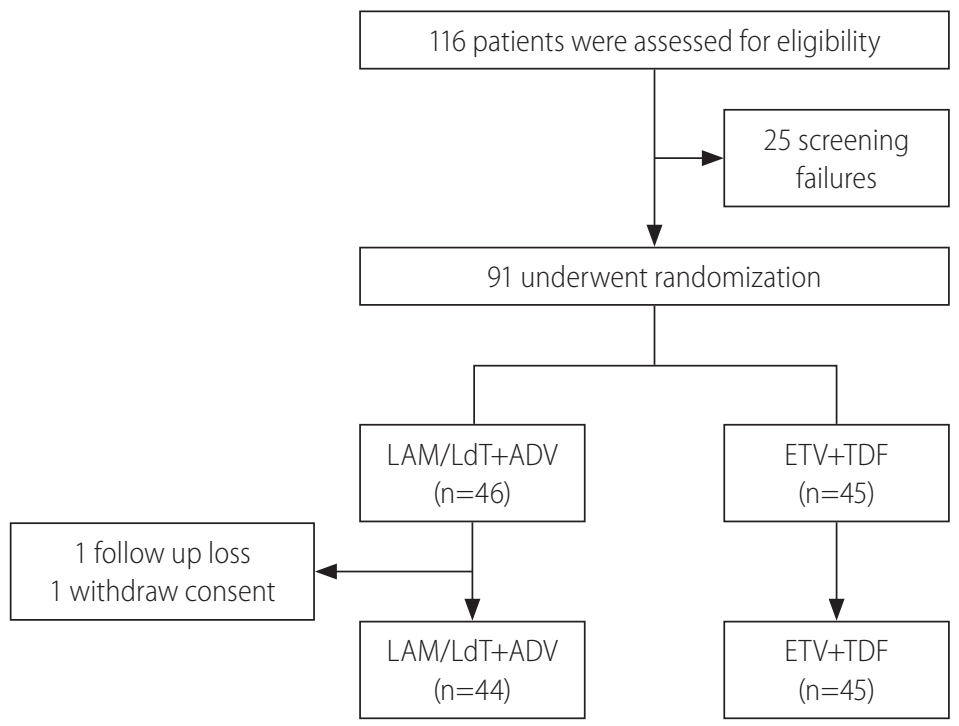

Figure 1. Study design. LAM, lamivudine; LdT, telbivudine; ADV, adefovir; ETV, entecavir; TDF, tenofovir.

Table 1. Baseline characteristics of the two study groups

\begin{tabular}{|c|c|c|c|}
\hline & LAM/LdT+ADV $(n=46)$ & ETV+TDF $(n=45)$ & $P$-value \\
\hline Age (years) & $50.0 \pm 10.5$ & $50.0 \pm 11.0$ & 0.978 \\
\hline Sex (male) & $28(60.9)$ & $31(68.9)$ & 0.512 \\
\hline Liver cirrhosis & $9(19.5)$ & $6(13.3)$ & 0.574 \\
\hline Duration of prior NUC treatment (weeks) & $307.9 \pm 116.8$ & $327.7 \pm 113.4$ & 0.536 \\
\hline Duration of ADV-based treatment (weeks) & $166.8 \pm 82.3$ & $173.1 \pm 85.3$ & 0.934 \\
\hline Prior use of ETV & $11(23.9)$ & $16(35.6)$ & 0.257 \\
\hline $\log _{10} \mathrm{HBV}$ DNA $(\mathrm{IU} / \mathrm{mL})$ & $4.0 \pm 0.7$ & $4.3 \pm 0.9$ & 0.228 \\
\hline HBeAg positive & $43(93.5)$ & $40(88.9)$ & 0.485 \\
\hline $\mathrm{ALT}(\mathrm{IU} / \mathrm{L})$ & $32.0 \pm 22.0$ & $27.0 \pm 17.4$ & 0.422 \\
\hline AST (IU/L) & $28.0 \pm 12.7$ & $23.0 \pm 8.3$ & 0.285 \\
\hline Total bilirubin (mg/dL) & $0.7 \pm 0.3$ & $0.7 \pm 0.2$ & 0.952 \\
\hline Albumin $(\mathrm{g} / \mathrm{dL})$ & $4.6 \pm 0.2$ & $4.6 \pm 0.3$ & 0.634 \\
\hline Calcium (mg/dL) & $9.3 \pm 0.3$ & $9.3 \pm 0.3$ & 0.850 \\
\hline Phosphate (mg/dL) & $3.4 \pm 0.7$ & $3.4 \pm 0.5$ & 0.613 \\
\hline Creatinine (mg/dL) & $0.8 \pm 0.2$ & $0.8 \pm 0.1$ & 0.718 \\
\hline $\mathrm{eGFR}\left(\mathrm{mL} / \mathrm{min} / 1.73 \mathrm{~m}^{2}\right)$ & $42.0 \pm 20.5$ & $42.0 \pm 20.5$ & 0.889 \\
\hline
\end{tabular}

Values are presented as mean \pm standard deviation or number (\%).

LAM, lamivudine; LdT, telbivudine; ADV, adefovir; ETV, entecavir; TDF, tenofovir; NUC, nucleos(t)ides; HBV, hepatitis B virus; HBeAg, hepatitis B e antigen; ALT, alanine aminotransferase; AST, aspartate aminotransferase; eGFR, Estimated glomerular filtration rate. 
Table 2. Prior nucleos(t)ide treatment regimens in the two study groups

\begin{tabular}{|c|c|c|c|}
\hline & LAM/LdT+ADV & ETV+TDF & $P$-value \\
\hline \multicolumn{4}{|l|}{ Prior regimen } \\
\hline No ETV use & & & 0.499 \\
\hline LAM & $21(45.7)$ & $13(28.9)$ & \\
\hline LdT & $2(4.3)$ & $0(0.0)$ & \\
\hline CLV & $0(0.0)$ & $1(2.2)$ & \\
\hline LAM, ADV & $10(21.7)$ & $9(20)$ & \\
\hline CLV, ADV & $1(2.2)$ & $3(6.7)$ & \\
\hline LAM, CLV & $0(0.0)$ & $1(2.2)$ & \\
\hline LAM, ADV, LdT & $1(2.2)$ & $2(4.4)$ & \\
\hline ETV use & $11(23.9)$ & $16(35.6)$ & \\
\hline LAM, ETV & $5(10.9)$ & $6(13.3)$ & \\
\hline CLV, ETV & $0(0.0)$ & $1(2.2)$ & \\
\hline ADV, ETV & $0(0.0)$ & $1(2.2)$ & \\
\hline PegIFN, ETV & $1(2.2)$ & $0(0.0)$ & \\
\hline LAM, ADV, ETV & $5(10.9)$ & $5(11.1)$ & \\
\hline LAM, ETV, LdT & $0(0.0)$ & $1(2.2)$ & \\
\hline ETV, ADV, LdT & $0(0.0)$ & $1(2.2)$ & \\
\hline LAM, ADV, ETV, LdT & $0(0.0)$ & $1(2.2)$ & \\
\hline \multicolumn{4}{|l|}{ Treatment at baseline } \\
\hline LAM+ADV & $30(65.2)$ & $28(62.2)$ & 0.829 \\
\hline LAM+LdT & $16(34.8)$ & $17(37.8)$ & \\
\hline
\end{tabular}

Values are presented as number (\%).

LAM, lamivudine; LdT, telbivudine; ADV, adefovir; ETV, entecavir; TDF, tenofovir; CLV, clevudine; PegIFN, peginterferon.

\section{Virologic response}

The proportion of patients who achieved virologic response (HBV DNA level $<60 \mathrm{IU} / \mathrm{mL}$ ) at week 48 was significantly higher in ETV+TDF group than in LAM/LdT+ADV group $(93.33 \%$ vs. $6.52 \%, P<0.001)$. ETV+TDF group also had a significantly greater cumulative virologic response at week 12 (73.33\% vs. 6.52\%), week $24(82.22 \%$ vs. $4.34 \%)$, week $36(88.89 \%$ vs. $4.34 \%)$, and week 48 (93.33\% vs. 6.52\%) (log-rank-test: $P<0.001$ for all comparisons; Fig. 2). A total of $91.3 \%$ of patients who continued LAM/LdT+ADV treatment had virologic nonresponse (defined as a $<1 \log _{10} \mathrm{IU} / \mathrm{mL}$ reduction in HBV DNA concentration from baseline at week 24 ) at week 24 , and $86.9 \%$ did not have a virologic response (HBV DNA level $\geq 60 \mathrm{IU} / \mathrm{mL}$ ) at week 48 . A total of $7 \%$ (3/45) of the patients who received ETV+TDF did not have a virologic response at week 48. These patients were all HBeAg-positive males who received previous ETV treatment. Their mean HBV DNA level was $5.93 \log _{10} \mathrm{IU} / \mathrm{mL}$ at baseline, $4.63 \log _{10} \mathrm{IU} / \mathrm{mL}$ at week 12, $4.31 \log _{10} \mathrm{IU} / \mathrm{mL}$ at week $24,3.97 \log _{10} \mathrm{IU} / \mathrm{mL}$ at week 36 , and $3.07 \log _{10} \mathrm{IU} / \mathrm{mL}$ at week 48 . A study of baseline mutations in reverse transcriptase (RT) indicated that one patient had rt180 (Met>Leu)+rt204(Val>Met)+rt184(Leu>Thr), one patient had rt204 (lle>Met), and one patient had rt180 (Met>Leu)+ rt204(Val>Met). These mutations were still present, and additional mutations did not develop until week 48 . The mean time to

Table 3. HBV reverse transcriptase mutations of patients in the two study groups

\begin{tabular}{|c|c|c|c|}
\hline Mutation(s) & LAM/LdT+ADV & ETV+TDF & $P$-value \\
\hline None & $1(2.2)$ & $2(4.4)$ & 0.603 \\
\hline rt204(Ile $\rightarrow$ Met $)$ & $16(34.8)$ & $10(22.2)$ & \\
\hline rt180 (Met $\rightarrow$ Leu) & $1(2.2)$ & $0(0.0)$ & \\
\hline rt180 (Met $\rightarrow$ Leu)+rt204(Val $\rightarrow$ Met) & $13(28.3)$ & $10(22.2)$ & \\
\hline rt180 (Met $\rightarrow$ Leu)+rt204(Ile $\rightarrow$ Met) & $9(19.6)$ & $12(26.7)$ & \\
\hline rt180 (Met $\rightarrow$ Leu)+rt204(Ile $\rightarrow$ Met)+rt204(Val $\rightarrow$ Met) & $2(4.3)$ & $5(11.1)$ & \\
\hline rt180 (Met $\rightarrow$ Leu)+rt204(Val $\rightarrow$ Met)+rt202(Gly $\rightarrow$ Ser) & $1(2.2)$ & $2(4.4)$ & \\
\hline rt180 (Met $\rightarrow$ Leu)+rt204(Val $\rightarrow$ Met)+rt184(Leu $\rightarrow$ Thr $)$ & $1(2.2)$ & $1(2.2)$ & \\
\hline rt180 (Met $\rightarrow$ Leu)+rt204(Val $\rightarrow$ Met)+rt184(Ser/Met $\rightarrow$ Thr) & $0(0.0)$ & $1(2.2)$ & \\
\hline rt180 (Met $\rightarrow$ Leu)+rt204(Ile $\rightarrow$ Met)+rt204(Val $\rightarrow$ Met)+rt184(Ser/Met $\rightarrow$ Thr) & $0(0.0)$ & $1(2.2)$ & \\
\hline rt180 (Met $\rightarrow$ Leu)+rt204(Val $\rightarrow$ Met)+rt184(Leu $\rightarrow$ Thr)+rt202(Gly $\rightarrow$ Ser) & $1(2.2)$ & $0(0.0)$ & \\
\hline rt180 (Met $\rightarrow$ Leu $)+r t 204($ Val $\rightarrow$ Met)+rt184(Leu $\rightarrow$ Thr $)+r t 250($ Val $\rightarrow$ Met $)$ & $0(0.0)$ & $1(2.2)$ & \\
\hline rt180 (Met $\rightarrow$ Leu)+rt204(Val $\rightarrow$ Met)+rt184(Ile/Ala $\rightarrow$ Thr)+rt202(Gly $\rightarrow$ Ser) & $1(2.2)$ & $0(0.0)$ & \\
\hline
\end{tabular}

Values are presented as number (\%).

LAM, lamivudine; LdT, telbivudine; ADV, adefovir; ETV, entecavir; TDF, tenofovir; lle, isoleucine; Met, methionine; Leu, leucine; Val, valine; Ser, serine; Thr, threonine; Gly, glycine. 


\section{CLINCAL and MOLECULAR}

virologic response was 18.33 weeks in ETV+TDF group and 43.13 weeks in LAM/LdT+ADV group.

Our analysis of the changes in viral load over time (Fig. 3) indicated that the mean $\log _{10}$ HBV DNA level (IU/mL) in the two groups were similar at baseline (4.08 vs. $4.36 \mathrm{IU} / \mathrm{mL}$ ). However, TDF+ETV had significantly greater decreases at weeks $12,24,36$, and 48 ( $P<0.001$ for all comparisons).

Univariate and multivariate analyses were performed to identify the factors associated with virologic response at week 48 (Table 4). Factors assessed in univariate analyses included treatment method (LAM/LdT+ADV vs. TDF+ETV), age, sex, presence of liver cir-

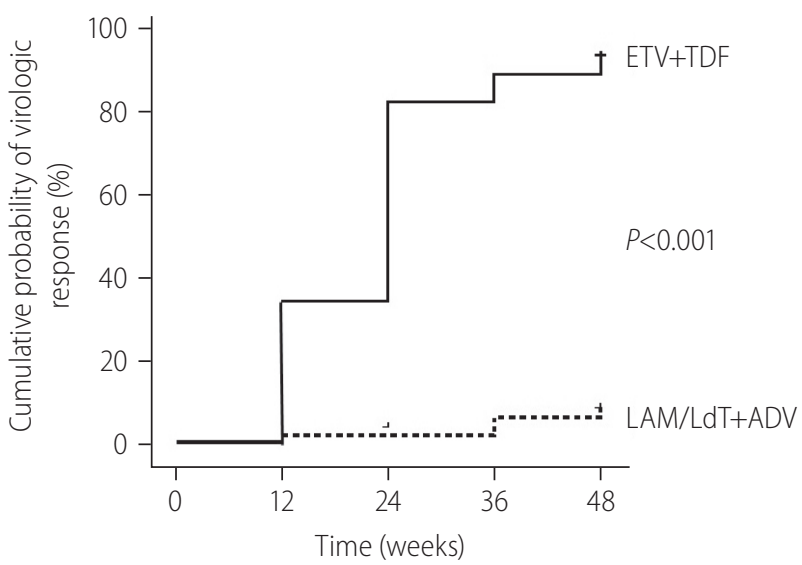

Figure 2. Cumulative virologic responses in the two groups. Solid line: TDF+ETV group; dotted line: LAM/LdT+ADV group. ETV, entecavir; TDF, tenofovir; LAM, lamivudine; LdT, telbivudine; ADV, adefovir. rhosis, overall duration of previous NUC treatment, duration of previous ADV treatment, serum baseline HBV DNA concentration, HBeAg positivity, prior use of ETV, baseline resistance, and ALT level. Multivariate analysis showed that treatment with TDF+ETV and low baseline HBV DNA concentration were significantly and positively associated with virologic response.

We performed the same analyses separately for each treatment group (data not shown). Only the patients in LAM/LdT+ADV group who achieved virologic response had low baseline HBV DNA levels ( $<4$ vs. $\geq 4 \log _{10} \mathrm{IU} / \mathrm{mL}: 21.1 \%$ [4/19] vs. $0 \%$ [0/26], $P=0.026)$. Patients in ETV+TDF group were less likely to achieve a

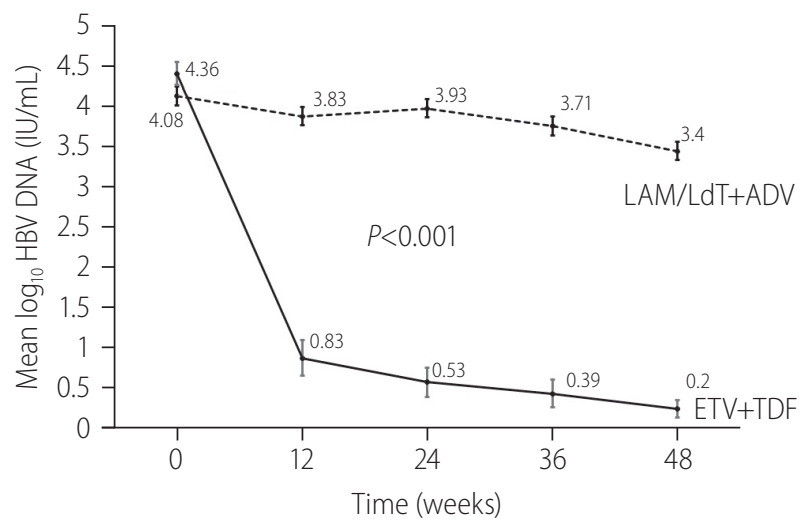

Figure 3. Reduction of HBV DNA level (mean $\log _{10} \mathrm{IU} / \mathrm{mL}$ ) in the two groups from baseline to week 48. Solid line: TDF+ETV group; dotted line: LAM/LdT+ADV group. HBV, hepatitis B virus; LAM, lamivudine; LdT, telbivudine; ADV, adefovir; ETV, entecavir; TDF, tenofovir.

Table 4. Univariate and multivariate analyses of the association of clinical factors with virologic response at week 48

\begin{tabular}{|c|c|c|c|c|c|c|}
\hline & \multicolumn{3}{|c|}{ Univariate analysis } & \multicolumn{3}{|c|}{ Multivariate analysis } \\
\hline & HR & $95 \% \mathrm{Cl}$ & $P$-value & HR & $95 \% \mathrm{Cl}$ & $P$-value \\
\hline Age & 0.992 & $0.954-1.031$ & 0.667 & & & \\
\hline Sex, male/female & 1.181 & $0.495-2.819$ & 0.708 & & & \\
\hline Liver cirrhosis & 1.714 & $0.555-5.297$ & 0.349 & & & \\
\hline Duration of prior NUC treatment & 0.995 & $0.981-1.010$ & 0.526 & & & \\
\hline Duration of ADV-based treatment & 0.995 & $0.975-1.015$ & 0.621 & & & \\
\hline $\log _{10} \mathrm{HBV}$ DNA & 1.172 & $0.721-1.906$ & 0.523 & 11.591 & $2.112-63.613$ & 0.005 \\
\hline HBeAg positivity & 6.450 & $0.744-55.948$ & 0.091 & 36.618 & $0.518-2,586.962$ & 0.097 \\
\hline ALT & 1.017 & $0.993-1.041$ & 0.157 & 1.019 & $0.978-1.061$ & 0.373 \\
\hline Prior use of ETV & 0.857 & $0.344-2.137$ & 0.742 & & & \\
\hline Treatment method, TDF+ETV vs. LAM/LdT+ADV & 0.007 & $0.001-0.033$ & 0.001 & 0.001 & $0.001-0.012$ & 0.001 \\
\hline Baseline genotypic resistance & 1.232 & $0.968-1.569$ & 0.090 & 1.245 & $0.715-2.169$ & 0.439 \\
\hline
\end{tabular}

HR, hazard ratio; Cl, confidence interval; NUC, nucleos(t)ide; ADV, adefovir; HBV, hepatitis B virus; HBeAg, hepatitis B e antigen; ALT, alanine aminotransferase; ETV, entecavir; TDF, tenofovir; LAM, lamivudine; LdT, telbivudine. 
virologic response if they had high baseline HBV DNA levels $(<5$ vs. $\geq 5 \log _{10} \mathrm{IU} / \mathrm{mL}: 100 \%$ [36/36] vs. $66.7 \%$ [6/9], $\left.P=0.006\right)$. None of the other baseline clinical factors were associated with virologic response in this subgroup analysis.

\section{Resistance surveillance}

We collected serum samples from baseline to week 48 in all patients. For patients with detectable serum HBV DNA, we genotypically analyzed the samples for mutations in HBV RT that are associated with resistance to LAM, ADV, and ETV (Table 5). Among those with one or more RT mutations associated with ETV resistance at baseline, 16.6\% (1/6) in ETV+TDF group and 75\% $(3 / 4)$ in LAM/LdT+ADV group retained these mutations until week $48(P=0.106)$. Among those who did not have RT mutations associated with ETV resistance at baseline, two of 39 patients in ETV+TDF group developed RT mutations associated with ETV resistance (one at week 12 and one at week 24). In LAM/LdT+ADV group, two of 42 patients developed mutations associated with ETV resistance (one at week 36 and one at week 48). Among patients with ETV resistance at baseline or during treatment, three patients in ETV+TDF group (one at baseline, one at week 12, one at week 24) and two patients in LAM/LdT+ADV group (one at week 36, one at week 48) were ETV-naïve. However, in ETV+TDF group, complete suppression was achieved in all but one patient with baseline ETV resistance mutations in RT after 48 weeks of ETV+TDF treatment. In LAM/LdT+ADV group, virologic response could not be achieved in three of four patients who had baseline RT mutations associated with ETV resistance, and additional mutations associated with ETV resistance developed even when continuing LAM/LdT+ADV treatment. We also analyzed the genotypic RT mutations associated with ADV resistance in all patients from baseline to week 48; a single patient in ETV+TDF group had such a mutation at week 24, but this mutation was no longer present at weeks 36 and 48, and this patient achieved virologic response following ETV+TDF treatment.

\section{Biochemical and serologic responses}

Five patients in ETV+TDF group and 11 patients in LAM/LdT+ ADV group had elevated ALT levels ( $\geq 40 \mathrm{IU} / \mathrm{mL}$ ) at baseline. After 48 weeks, the ALT level normalized in one patient $(20 \%)$ in ETV+TDF group and in two patients (18.2\%) in LAM/LdT+ADV group ( $P=1.000$, Kaplan-Meier method).

Forty patients in ETV+TDF group (88.9\%) and 44 patients (95.6\%) in LAM/LdT+ADV group were HBeAg-positive at baseline. Two patients (5\%) in ETV+TDF group approached HBeAg seroconversion, and one patient (2.3\%) in LAM/LdT+ADV group achieved HBeAg seroconversion $(P=0.606)$.

Table 5. Genotypic resistance to ETV (top) and ADV (bottom) from baseline to week 48 in the two study groups

\begin{tabular}{|c|c|c|c|}
\hline Mutation and date & LAM/LdT+ADV & ETV+TDF & $P$-value \\
\hline Resistance mutation to ETV at baseline & $4(8.7)$ & $6(13.3)$ & 0.522 \\
\hline Retention of baseline mutations at week 12 & $3(6.5)$ & $2(4.4)$ & 1.000 \\
\hline Additional emergence of mutations at week 12 & $0(0.0)$ & $1(2.2)$ & \\
\hline Retention of baseline mutations at week 24 & $3(6.5)$ & $2(4.4)$ & 1.000 \\
\hline Additional emergence of mutations at week 24 & $0(0.0)$ & $1(2.2)$ & \\
\hline Retention of baseline mutations at week 36 & $3(6.5)$ & $1(2.2)$ & 0.192 \\
\hline Additional emergence of mutations at week 36 & $1(2.1)$ & $0(0.0)$ & \\
\hline Retention of baseline mutations at week 48 & $3(6.5)$ & $1(2.2)$ & 0.106 \\
\hline Additional emergence of mutations at week 48 & $2(4.3)$ & $0(0.0)$ & \\
\hline Resistance mutation to ADV at baseline & $0(0.0)$ & $0(0.0)$ & \\
\hline Retention of baseline mutations at week 24 & $0(0.0)$ & $0(0.0)$ & 1.000 \\
\hline Additional emergence of mutations at week 24 & $0(0.0)$ & $1(2.2)$ & \\
\hline Retention of baseline mutations at week 48 & $0(0.0)$ & $0(0.0)$ & 1.000 \\
\hline Additional emergence of mutations at week 48 & $0(0.0)$ & $0(0.0)$ & \\
\hline
\end{tabular}

Values are presented as number (\%).

ETV, entecavir; ADV, adefovir; LAM, lamivudine; LdT, telbivudine; TDF, tenofovir. 


\section{Safety}

There were 35 adverse events in 23 patients (11 in LAM/LdT+ ADV group and 12 in ETV+TDF group) during the 48-week study period, and three of these events were related to the study drug (one patient with chest pain in LAM/LdT+ADV group, two patients with nausea and dyspepsia in ETV+TDF group, $P=0.762$ ). Two patients in ETV+TDF group (one with unstable angina, one with headache) and two patients in LAM/LdT+ADV group (both with $\mathrm{HCC}$ ) discontinued therapy due to severe adverse events. However, none of these severe adverse events were related to the study drugs, and these patients improved after receiving medical treatment. None of the patients experienced elevated serum creatinine $(\geq 0.5 \mathrm{mg} / \mathrm{dL})$, decreased serum phosphorus $(\leq 3 \mathrm{mg} / \mathrm{dL})$, or serum ALT flare ( $>10$-fold above the ULN) during the study period.

\section{DISCUSSION}

The results of this trial of patients with LAM-resistant HBV who showed suboptimal response to LAM/LdT+ADV combination therapy clearly showed that treatment with ETV+TDF provided significantly more suppression of HBV than did treatment with LAM/ LdT+ADV. Moreover, continuation of LAM/LdT+ADV treatment provided little antiviral benefit, and also increased the emergence of additional RT mutations that confer resistance to ADV.

Previous studies have recommended LAM/LdT+ADV combination therapy as a treatment option for patients with LAM-resistant HBV infections. ${ }^{3-5}$ This combination therapy can prevent additional development of ADV-resistant mutations. ${ }^{13,26}$ However, as the use of continued LAM has no effect on virologic response in patients with LAM-resistant HBV, the combination of LAM/LdT+ADV provides no increased antiviral efficacy relative to ADV monotherapy. ${ }^{13,14}$ Furthermore, ADV has modest potency in suppressing HBV DNA replication, ${ }^{3}$ and a substantial proportion of patients have shown inadequate or suboptimal virologic responses during treatment with LAM/LdT+ADV. ${ }^{13,27}$

Following the introduction of ETV and TDF, numerous studies have assessed the antiviral efficacy of ETV alone, ETV+ADV, TDF alone, and TDF+ETV in patients with antiviral drug-resistant $\mathrm{HBV}^{22,28-31}$ ETV monotherapy is not optimal for the treatment of $L A M$-refractory HBV, as it leads to a lower virologic response rate in patients with LAM-resistant HBV than in LAM-naïve patients, and LAM-resistant HBV has partial resistance to ETV. ${ }^{28,29}$ In addi- tion, genotypic resistance to ETV frequently emerges during longterm treatment of patients with LAM resistance. ${ }^{29,32}$ Prior studies showed that ETV+ADV led to better antiviral efficacy than did LAM+ADV. ${ }^{25}$ Furthermore, TDF-containing regimens had better antiviral efficacy compared to ETV+ADV. ${ }^{23}$ Recent studies showed that TDF monotherapy provided a virologic response comparable to that of TDF+ETV combination therapy, even in patients with ADV-resistant HBV and multiple-drug failures. ${ }^{33,34}$

Since none of the patients in this study was treated with TDF monotherapy, efficacy and safety could not be compared between patients who were treated with TDF and those treated with TDF+ETV. The efficacy of TDF was shown to be non-inferior to that of TDF+ETV, with TDF monotherapy associated with lower costs and lower risks of adverse events compared to TDF+ETV. ${ }^{33-35}$ Nevertheless, antiviral resistance can emerge despite resistance to TDF having a very high barrier. ${ }^{36}$ In this study, baseline ETV resistance remained even after 48 weeks of TDF+ETV combination therapy. Since patients with $\mathrm{CHB}$ require long-term antiviral therapy, resistance to highly potent antiviral agents could develop if HBV quasispecies perpetually evolve and acquire drug-resistant mutations under pressure from these antiviral agents. ${ }^{36,37}$ At that point, long-term TDF+ETV combination therapy might be a better option than long-term TDF monotherapy. To our knowledge, this is the first prospective study of patients who had LAM-resistant HBV and suboptimal responses to LAM/LdT+ADV to directly compare the efficacy of switching to ETV+TDF and continuing LAM/ LdT+ADV.

This study found that the DNA suppression rate at week 48 with ETV+TDF treatment was $93.33 \%$. This rate was higher than that observed when LAM-resistant patients were treated with ETV+TDF (84.8\%), ${ }^{23}$ and much higher than that observed in patients infected with HBV variants that were resistant to LAM and ETV in a real-world study (51.8\%). ${ }^{35}$ These previous studies, however, were retrospective in design. Therefore, this study is the first randomized prospective trial of South Korean patients who had LAM-resistant HBV infections and suboptimal responses to LAM/ LdT+ADV.

The reason for the higher virologic response observed in the present study is probably that we excluded patients with ADV resistance when enrolling the patients, as one of the treatment arms was LAM+ADV. It has been shown that the response to $L A M+A D V$ is greatly reduced in patients with high viral loads and mutations, causing resistance to both drugs (e.g., rtA181V/T with or without rtN236T) at the initiation of treatment. ${ }^{27,38}$ However, $57.1 \%$ of the enrolled patients changed antiviral agents more than 
three times before our LAM/LdT+ADV regimen; $29.7 \%$ received ETV treatment and $8.7 \%$ had mutations associated with ETV resistance and LAM resistance. Therefore, the efficacy of TDF+ETV treatment in our patients could be considered excellent compared to previous studies. The excellent virologic response to 48 weeks of TDF+ETV combination therapy in this study suggests that combination therapy could be a better option for patients with suboptimal response to $L A M / L d T+A D V$ for the prevention of further resistance. More specifically, 48 weeks of TDF+ETV combination therapy was highly effective in rapidly reducing the serum HBV DNA level to below the detection limit of real-time PCR, and thereby preventing the development of resistance.

Importantly, we found that the continuation of LAM/LdT+ADV treatment in patients with suboptimal responses provided little antiviral benefits to patients who had LAM-resistant HBV, and also promoted the emergence of multidrug-resistant strains of HBV. In fact, $91.3 \%$ of patients who continued on LAM/LdT+ADV experienced virologic nonresponse at week 24 , and $86.9 \%$ did not experience virologic response at week 48. Furthermore, threequarters of the patients in LAM/LdT+ADV group who had baseline ETV resistance did not achieve virologic response, and $4.7 \%$ (2/42) of patients also developed additional RT mutations responsible for ETV resistance while receiving LAM/LdT+ADV. However, with the exception of one patient with baseline ETV resistance mutation, all of the patients who were treated for 48 weeks with ETV+TDF combination treatment showed complete virologic suppression. These findings suggest that ETV+TDF combination treatment can provide virologic suppression, even if there are mutations other than those that cause LAM resistance. A previous study reported that 15\% (5/33) of HBV patients without mutations that were associated with ADV resistance at baseline developed ADV resistance mutations at week 52 during continuous LAM/LdT+ADV treatment. ${ }^{25}$ We found no additional ADV resistance in LAM/LdT+ADV group, probably because we had excluded patients with ADV resistance at baseline.

Our patients generally exhibited good tolerance to each drug combination during the 48-week treatment period. No patient required dose reduction or discontinuation due to a drug-related adverse event. There were also no significant changes in serum creatinine and serum phosphorus concentrations.

This study had some limitations. First, the study duration was relatively short. Although ETV+TDF combination treatment showed excellent virologic suppression in patients with LAM-resistant HBV who had suboptimal responses to LAM/LdT+ADV, 7\% $(3 / 45)$ of the patients who received ETV+TDF still showed incom- plete virologic suppression at week 48. Since ETV resistance can develop several years later in patients with LAM resistance, the duration of this study (48 weeks) was insufficient to examine this effect. Second, this study had an open label design, with no placebo and no blinding. Although our endpoints were objective (virologic response and biochemical response) and were determined by laboratory tests, more objective measurements of adverse events could be achieved with blinding. Finally, a recent trial showed that TDF monotherapy was comparable to ETV+TDF combination therapy in patients infected with HBV variants that were resistant to $A D V$ and ETV. ${ }^{33,34}$ Therefore, it might be more informative to compare TDF monotherapy with ETV+TDF.

In summary, this study had three major conclusions. First, ETV+TDF led to a significantly higher rate of virologic response compared to LAM/LdT+ADV in patients with LAM-resistant HBV who had suboptimal responses to $L A M / L d T+A D V$ regardless of the HBV genotypic resistance profile. Second, the continuation of LAM/LdT+ADV provided very little antiviral benefit, and increased the risk of RT mutations that confer drug resistance. Third, the two treatment regimens had similar safety profiles. Therefore, we conclude that promptly switching to a more potent antiviral regimen should be considered for patients who have LAM-resistant HBV infections and suboptimal responses to LAM/LdT+ADV therapy, and that ETV+TDF combination treatment is a viable option.

\section{Authors' contribution}

Study design was by JH and SHA, data analysis and interpretation was by HYW, SHA and JH. Study write-up was by HYW. Enrolment, management of patients and data collection was by JYP, SHA, SHB, CWK, JYJ, WYT, DJK, IHK, JH. All authors had access to all data in the study and revised the article. JH, SHA and HYW made the final decision to submit the study for publication.

\section{Acknowledgements}

This investigator-initiated trial was supported by an unrestricted grant from Bristol-Myers Squibb (BMS) and clinical research grant from Pusan National University Hospital in 2018. BMS had no role in the study design and the authors retain full responsibility for the collection and interpretation of data, decision to publish, and preparation of the manuscript.

\section{Conflicts of Interest}

The authors have no conflicts to disclose. 


\section{REFERENCES}

1. Chen CJ, Yang HI, Su J, Jen CL, You SL, Lu SN, et al. Risk of hepatocellular carcinoma across a biological gradient of serum hepatitis $B$ virus DNA level. JAMA 2006;295:65-73.

2. Iloeje UH, Yang HI, Su J, Jen CL, You SL, Chen CJ, et al. Predicting cirrhosis risk based on the level of circulating hepatitis $B$ viral load. Gastroenterology 2006;130:678-86.

3. European Association For The Study Of The Liver. EASL clinical practice guidelines: management of chronic hepatitis B. J Hepatol 2009;50:227-242.

4. Liaw YF, Leung N, Kao JH, Piratvisuth T, Gane E, Han KH, et al. Asian-Pacific consensus statement on the management of chronic hepatitis B: a 2008 update. Hepatol Int 2008;2:263-283.

5. Lok AS, McMahon BJ. Chronic hepatitis B: update 2009. Hepatology 2009;50:661-662.

6. Gordon SC, Lamerato LE, Rupp LB, Li J, Holmberg SD, Moorman AC, et al. Antiviral therapy for chronic hepatitis $B$ virus infection and development of hepatocellular carcinoma in a US population. Clin Gastroenterol Hepatol 2014;12:885-893.

7. Eun JR, Lee HJ, Kim TN, Lee KS. Risk assessment for the development of hepatocellular carcinoma: according to on-treatment viral response during long-term lamivudine therapy in hepatitis B virusrelated liver disease. J Hepatol 2010;53:118-125.

8. Papatheodoridis GV, Dimou E, Dimakopoulos K, Manolakopoulos $S$, Rapti I, Kitis $G$, et al. Outcome of hepatitis B e antigen-negative chronic hepatitis B on long-term nucleos(t)ide analog therapy starting with lamivudine. Hepatology 2005;42:121-129.

9. Yuen MF, Seto WK, Chow DH, Tsui K, Wong DK, Ngai VW, et al. Long-term lamivudine therapy reduces the risk of long-term complications of chronic hepatitis B infection even in patients without advanced disease. Antivir Ther 2007;12:1295-1303.

10. Lok AS, McMahon BJ; Practice Guidelines Committee, American Association for the Study of Liver Diseases (AASLD). Chronic hepatitis B: update of recommendations. Hepatology 2004;39:857-861.

11. Lok AS, Lai CL, Leung N, Yao GB, Cui ZY, Schiff ER, et al. Long-term safety of lamivudine treatment in patients with chronic hepatitis $B$. Gastroenterology 2003;125:1714-1722.

12. Fung SK, Chae HB, Fontana RJ, Conjeevaram H, Marrero J, Oberhelman $K$, et al. Virologic response and resistance to adefovir in patients with chronic hepatitis B. J Hepatol 2006;44:283-290.

13. Rapti I, Dimou E, Mitsoula P, Hadziyannis SJ. Adding-on versus switching-to adefovir therapy in lamivudine-resistant HBeAg-negative chronic hepatitis B. Hepatology 2007;45:307-313.

14. Peters MG, Hann HW, Martin P, Heathcote EJ, Buggisch P, Rubin R, et al. Adefovir dipivoxil alone or in combination with lamivudine in patients with lamivudine-resistant chronic hepatitis B. Gastroenterology 2004;126:91-101.
15. Fung J, Lai CL, Yuen JC, Wong DK, Tanaka Y, Mizokami M, et al. Adefovir dipivoxil monotherapy and combination therapy with lamivudine for the treatment of chronic hepatitis B in an Asian population. Antivir Ther 2007;12:41-46.

16. Lampertico P. Partial virological response to nucleos(t)ide analogues in naïve patients with chronic hepatitis $B$ : from guidelines to field practice. J Hepatol 2009;50:644-647.

17. Santantonio T, Fasano M, Durantel S, Barraud L, Heichen M, Guastadisegni A, et al. Adefovir dipivoxil resistance patterns in patients with lamivudine-resistant chronic hepatitis B. Antivir Ther 2009;14:557-565.

18. Lim YS, Lee TH, Heo NY, Shim JH, Lee HC, Suh DJ. Entecavir plus adefovir combination treatment for chronic hepatitis B patients after failure of nucleoside/nucleotide analogues. Antivir Ther 2012;17:5360.

19. Petersen J, Ratziu V, Buti M, Janssen HL, Brown A, Lampertico P, et al. Entecavir plus tenofovir combination as rescue therapy in pretreated chronic hepatitis B patients: an international multicenter cohort study. J Hepatol 2012;56:520-526.

20. Patterson SJ, George J, Strasser SI, Lee AU, Sievert W, Nicoll AJ, et al. Tenofovir disoproxil fumarate rescue therapy following failure of both lamivudine and adefovir dipivoxil in chronic hepatitis B. Gut 2011;60:247-254.

21. Shim JH, Suh DJ, Kim KM, Lim YS, Lee HC, Chung YH, et al. Efficacy of entecavir in patients with chronic hepatitis $B$ resistant to both lamivudine and adefovir or to lamivudine alone. Hepatology 2009;50:1064-1071.

22. van Bömmel F, de Man RA, Wedemeyer H, Deterding K, Petersen J, Buggisch $P$, et al. Long-term efficacy of tenofovir monotherapy for hepatitis B virus-monoinfected patients after failure of nucleoside/ nucleotide analogues. Hepatology 2010;51:73-80.

23. Park JG, Park SY. Entecavir plus tenofovir versus entecavir plus adefovir in chronic hepatitis B patients with a suboptimal response to lamivudine and adefovir combination therapy. Clin Mol Hepatol 2015;21:242-248.

24. Han KH, Hong SP, Choi SH, Shin SK, Cho SW, Ahn SH, et al. Comparison of multiplex restriction fragment mass polymorphism and sequencing analyses for detecting entecavir resistance in chronic hepatitis B. Antivir Ther 2011;16:77-87.

25. Lim YS, Lee JY, Lee D, Shim JH, Lee HC, Lee YS, et al. Randomized trial of entecavir plus adefovir in patients with lamivudine-resistant chronic hepatitis B who show suboptimal response to lamivudine plus adefovir. Antimicrob Agents Chemother 2012;56:2941-2947.

26. Lampertico P, Viganò $M$, Manenti $E$, lavarone $M$, Sablon E, Colombo $M$. Low resistance to adefovir combined with lamivudine: a 3-year study of 145 lamivudine-resistant hepatitis B patients. Gastroenterology 2007;133:1445-1451.

27. Lampertico P, Viganò M, Manenti E, lavarone M, Lunghi G, Colombo 
M. Adefovir rapidly suppresses hepatitis B in HBeAg-negative patients developing genotypic resistance to lamivudine. Hepatology 2005;42:1414-1419.

28. Chang TT, Gish RG, Hadziyannis SJ, Cianciara J, Rizzetto M, Schiff $E R$, et al. A dose-ranging study of the efficacy and tolerability of entecavir in lamivudine-refractory chronic hepatitis B patients. Gastroenterology 2005;129:1198-1209.

29. Sherman M, Yurdaydin C, Simsek H, Silva M, Liaw YF, Rustgi VK, et al. Entecavir therapy for lamivudine-refractory chronic hepatitis B: improved virologic, biochemical, and serology outcomes through 96 weeks. Hepatology 2008;48:99-108.

30. Kim YJ, Sinn DH, Gwak GY, Choi MS, Koh KC, Paik SW, et al. Tenofovir rescue therapy for chronic hepatitis B patients after multiple treatment failures. World J Gastroenterol 2012;18:6996-7002.

31. Lee Cl, Kwon SY, Kim JH, Choe WH, Lee CH, Yoon EL, et al. Efficacy and safety of tenofovir-based rescue therapy for chronic hepatitis $B$ patients with previous nucleo(s/t)ide treatment failure. Gut Liver 2014;8:64-69.

32. Tenney DJ, Rose RE, Baldick CJ, Pokornowski KA, Eggers BJ, Fang $J$, et al. Long-term monitoring shows hepatitis $B$ virus resistance to entecavir in nucleoside-naïve patients is rare through 5 years of therapy. Hepatology 2009;49:1503-1514.
33. Lim YS, Byun KS, Yoo BC, Kwon SY, Kim YJ, An J, et al. Tenofovir monotherapy versus tenofovir and entecavir combination therapy in patients with entecavir-resistant chronic hepatitis B with multiple drug failure: results of a randomised trial. Gut 2016;65:852-860.

34. Lim YS, Yoo BC, Byun KS, Kwon SY, Kim YJ, An J, et al. Tenofovir monotherapy versus tenofovir and entecavir combination therapy in adefovir-resistant chronic hepatitis B patients with multiple drug failure: results of a randomised trial. Gut 2016;65:1042-1051.

35. Jeon HJ, Jung SW, Park NH, Yang Y, Noh JH, Ahn JS, et al. Efficacy of tenofovir-based rescue therapy for chronic hepatitis $B$ patients with resistance to lamivudine and entecavir. Clin Mol Hepatol 2017;23:230-238.

36. Liang $L Y$, Wong GL. Unmet need in chronic hepatitis B management. Clin Mol Hepatol 2019;25:172-180.

37. Lee $Y B$, Lee JH. Is tenofovir monotherapy a sufficient defense line against multi-drug resistant hepatitis B virus? Clin Mol Hepatol 2017;23:219-221.

38. Buti M, Elefsiniotis I, Jardi R, Vargas V, Rodriguez-Frias F, Schapper $M$, et al. Viral genotype and baseline load predict the response to adefovir treatment in lamivudine-resistant chronic hepatitis B patients. J Hepatol 2007;47:366-372. 